\title{
Etoposide Combined with FLAG Salvage Therapy Is Effective in Multiple Relapsed/Refractory Acute Myeloid leukemia
}

\author{
Jonas Westhus $^{\mathrm{a}} \quad$ Richard Noppeney $^{\mathrm{a}}$ Christine Schmitz $^{\mathrm{a}} \quad$ Michael Flasshove $^{\mathrm{b}}$ \\ Ulrich Dührsen $^{\mathrm{a}}$ Maher Hanoun ${ }^{\mathrm{a}}$

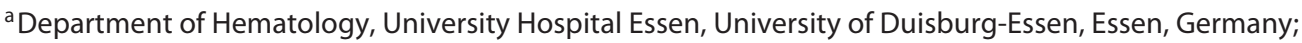 \\ ${ }^{b}$ Department of Hematology/Oncology, Düren Hospital, Düren, Germany
}

\section{Keywords}

Acute myeloid leukemia - Salvage therapy · FLAG .

Etoposide

\begin{abstract}
Managing acute myeloid leukemia (AML) is often hampered by repeated failure to achieve complete remission as well as recurrent relapse that causes an emergent need for alternative salvage therapies. The efficacy of most salvage therapies is based on anthracycline combinations. In highly pretreated patients who are not eligible for anthracycline-based protocols therapeutic alternatives are limited. For this particular group we evaluated the efficacy and safety of fludarabine, cytarabine, granulocyte colony-stimulating factor (FLAG) in combination with etoposide (FLAG-Eto) in 36 patients. The complete remission rate (CR) was $25.7 \%$ with a median overall survival of 6 months ( $95 \% \mathrm{Cl} 4.5-7.7)$. The median diseasefree survival for CR/CRi/MLFS (CR/CR with incomplete hematological recovery/morphologic leukemia-free state) patients was 8 months $(95 \% \mathrm{Cl} 0.6-15.5)$. The mortality rate on day 30 was $8 \%$ and increased on day 60 to $17 \%$. Our results show meaningful anti-leukemic activity of the FLAG-Eto reg-
\end{abstract}

karger@karger.com www.karger.com/aha

(C) 2019 S. Karger AG, Base

Karger" imen with a moderate toxicity profile in heavily pretreated relapsed/refractory AML patients enabling consolidating allogeneic stem cell transplantation.

(c) 2019 S. Karger AG, Basel

\section{Introduction}

Over decades no significant increase in the therapeutic response in acute myeloid leukemia (AML) could be documented [1], while improvement in outcome, in particular for younger patients, is more likely due to improved supportive care. Only recently, a number of targeted compounds have shown significant improvement in response rates with meaningful, but not substantial improvement in long-term outcome in AML [2]. Relapse or refractory disease is still a frequently occurring issue in AML. For relapsed/refractory AML patients the current curative treatment approach usually includes anthracycline-based re-induction chemotherapy to achieve deep remission followed by consolidating allogeneic stem cell transplantation. However, even with intensive salvage treatment protocols only half of the patients achieve re- 
mission with a median disease-free survival of only 7-17 months [3-5]. Therefore, salvage therapies are required in managing AML patients. Multiple relapsed/refractory AML patients, however, frequently have reached the maximum cumulative anthracycline doses and often show a compromised performance status which again hampers following consolidation therapies. Hence, welltolerable salvage therapies considering the requirements of heavily pretreated AML patients are urgently needed. Many salvage treatment protocols are based on the combination of fludarabine, cytarabine, and granulocyte stimulating factors, abbreviated to FLAG [6-8]. Recently, we have demonstrated the efficacy and safety of the FLAG protocol combined with idarubicin in a large patient cohort of primary refractory and first-time relapsed AML patients reaching overall complete remission rates $(\mathrm{CR}+$ $\mathrm{CR}$ with incomplete hematological recovery [CRi]) of $56 \%$ with a median overall survival of 15 months (95\% CI 5.7-25.1) [9]. For heavily pretreated patients who are not eligible for further anthracycline administration we now introduce another FLAG-based salvage therapy protocol combined with the topoisomerase II-inhibitor etoposide (FLAG-Eto). In this study, we analyzed the efficacy and toxicity profile of the FLAG-Eto regimen in this special patient population. This study finally paves the way for chemotherapy-based salvage treatment protocols tailored to the needs of different subsets of AML patients.

\section{Patients and Methods}

\section{Patients}

Thirty-six patients with relapsed/refractory AML had been treated according to the FLAG-Eto protocol between 1996 and 2016 and were retrospectively analyzed. Patients with AML M3 were not included. Cytogenetic risk groups were defined according to the Medical Research Council (MRC) criteria of Grimwade et al. [10], while complex aberrant karyotypes were defined as $\geq 3$ aberrations [11-14]. Preceding hematological disorders diagnosed at least 6 months before the diagnosis of AML were marked as secondary AML.

\section{Treatment Protocols}

The FLAG-Eto protocol consisted of fludarabine $\left(30 \mathrm{mg} / \mathrm{m}^{2} /\right.$ day) and cytarabine ( $2 \mathrm{~g} / \mathrm{m}^{2} /$ day) for 5 days, during which etoposide $\left(150 \mathrm{mg} / \mathrm{m}^{2} /\right.$ day) was administered on 3 days; granulocyte colony-stimulating factor (G-CSF) was given one day prior to chemotherapy for 6 days in cases where leukocytes were below $20 / \mathrm{nL}$.

\section{Safety and Response Evaluation}

Safety was retrospectively evaluated according to the established Common Terminology Criteria for Adverse Events (CTC$\mathrm{AE}$, version 4.0) [15]. Values of creatinine and liver enzymes were considered until day 28 after the last chemotherapy application.
For patients with elevated liver enzymes before starting therapy, only further elevations up to the next CTCAE grade were taken into account. Analysis of hematological recovery was only performed in patients achieving partial remission or better. Duration of neutropenia was defined as the latency to neutrophil recovery $\geq 0.5 / \mathrm{nL}$. The duration of hospitalization was calculated from the first day of therapy until discharge or in cases of continuous hospitalization until initiation of a new treatment, e.g., allogeneic transplantation. Response to treatment was assessed according to established remission criteria $[12,16]$. Two patients fulfilling all CR criteria but whose hematopoietic regeneration could not be retrospectively evaluated due to unavailability of related peripheral blood counts were therefore declared as CRi/MLFS (morphologic leukemia-free state). Of note, these 2 patients showed longterm survival over 200 months. Blast reduction was defined as at least $50 \%$ reduction of leukemic burden with maximum 5-25\% residual bone marrow blasts without regeneration of neutrophils $(<1 / \mathrm{nL})$ and/or platelets $(<100 / \mathrm{nL})$.

\section{Statistical Analyses}

Statistical analyses were performed by IBM SPSS Statistics Software version 22.0 (SPSS, Chicago, IL, USA). Overall survival was defined as duration from the first day of salvage therapy until death or alive at last follow-up (censored). Disease-free survival was only measured for CR/CRi/MLFS patients and was defined as duration from first day of documented remission after re-induction therapy until relapse, death (censored), or alive without relapse at last follow-up (censored). Overall survival and disease-free survival were estimated by Kaplan-Meier method. The log-rank test or log-rank test for trend was used to compare survival distributions in subgroups. Distributions of patient characteristics and response rates in subgroups were compared in the case of categorical variables by $\chi^{2}$ test, or if indicated by Fisher's exact test or linear-by-linear association. In the case of continuous variables and medians of ordinal variables the $t$ test or Mann-Whitney $\mathrm{U}$ test were used. All $p$ values were 2 -sided and were considered significant at a level $<0.05$.

\section{Results}

\section{Patient Characteristics}

In total, 36 patients received the FLAG-Eto protocol, of whom at least $91.7 \%$ completed the treatment. Dose modifications of at least $20 \%$ of one substance occurred in $25 \%$ of FLAG-Eto-treated patients. Table 1 presents patients' demographic and baseline characteristics. The median age was 57 years (range 28-71) with approximately $30 \%$ of patients over 60 years of age. All patients received intensive induction chemotherapy at initial diagnosis of AML. Median duration of last remission was 10.7 months (range 1.5-125.9 months). Early relapse within the first 12 months occurred in $64 \%(n=14 / 22)$ of patients; $16.7 \%$ of treated AML patients were primary refractory while almost all showed minor or no response to the first or second induction therapy. Strikingly, 36\% 
Table 1. Patient characteristics $(n=36)$

\begin{tabular}{|c|c|}
\hline & FLAG-Eto \\
\hline \multicolumn{2}{|l|}{ Age at relapse, years } \\
\hline Mean & $53 \pm 11$ \\
\hline Median & $57(28-71)$ \\
\hline \multicolumn{2}{|l|}{ Age at relapse } \\
\hline$\leq 60$ years & $25(69.4)$ \\
\hline$>60$ years & $11(30.6)$ \\
\hline \multicolumn{2}{|l|}{ Gender } \\
\hline Male & $18(50)$ \\
\hline Female & $18(50)$ \\
\hline \multicolumn{2}{|l|}{ Subtype } \\
\hline De novo & $29(80.6)$ \\
\hline Secondary & $7(19.4)$ \\
\hline Previous BMD & $1(2.8)$ \\
\hline Therapy-related & $6(16.7)$ \\
\hline \multicolumn{2}{|l|}{ Cytogenetic risk group at diagnosis } \\
\hline Favorable & $4(11.1)$ \\
\hline Intermediate & $23(63.9)$ \\
\hline Unfavorable & $7(19.4)$ \\
\hline Not available & $2(5.6)$ \\
\hline NPM1 mutation & $9(25.0)$ \\
\hline Missing & $18(50.0)$ \\
\hline FLT3-ITD mutation & $5(13.9)$ \\
\hline Missing & $18(50.0)$ \\
\hline CEBPA mutation & $1(2.8)$ \\
\hline Missing & $31(86.1)$ \\
\hline \multicolumn{2}{|l|}{ Blood counts at relapse } \\
\hline WBC, $1 / \mathrm{nL}$ & $4.30(0.38-94.13)$ \\
\hline Missing & $5(13.9)$ \\
\hline Plt, $1 / \mathrm{nL}$ & $39.0(1.0-427.0)$ \\
\hline Missing & $5(13.9)$ \\
\hline $\mathrm{Hb}, \mathrm{g} / \mathrm{dL}$ & $10.10(7.40-15.80)$ \\
\hline Missing & $5(13.9)$ \\
\hline $\mathrm{LDH}, \mathrm{U} / \mathrm{L}$ & $371(92-4,562)$ \\
\hline Missing & $5(13.9)$ \\
\hline Blasts in BM at relapse, $\%$ & $45(10-91)$ \\
\hline Missing & $15(41.7)$ \\
\hline Median ECOG PS at relapse & $1(0-4)$ \\
\hline \multicolumn{2}{|l|}{ ECOG PS at relapse } \\
\hline$\leq 2$ & $21(58.3)$ \\
\hline$>2$ & $4(11.1)$ \\
\hline Not available & $11(30.6)$ \\
\hline Median CCI at relapse & $0(0-5)$ \\
\hline \multicolumn{2}{|l|}{ CCI at relapse } \\
\hline 0 & $27(75.0)$ \\
\hline 1 & $3(8.3)$ \\
\hline 2 & $4(11.1)$ \\
\hline 3 & $0(0.0)$ \\
\hline 4 & $1(2.8)$ \\
\hline 5 & $1(2.8)$ \\
\hline \multicolumn{2}{|l|}{ Disease status ${ }^{\mathrm{a}}$} \\
\hline PIF & $6(16.7)$ \\
\hline 1st relapse & $17(47.2)$ \\
\hline$\geq 2$ nd relapse & $13(36.1)$ \\
\hline Median duration of last CR/CRi ${ }^{\mathrm{b}}$, months & $10.74(1.48-125.93)$ \\
\hline
\end{tabular}

$\begin{array}{lr}\text { Duration of last CR/CRi } & \\ \quad \leq 12 \text { months } & 14 / 22(63.6) \\ >12 \text { months } & 8 / 22(36.4) \\ \text { Median prior relapse therapies }{ }^{\mathrm{a}}, n & 1(0-2) \\ \text { Prior relapse therapies }^{\mathrm{a}} & \\ 0 & 13(36.1) \\ 1 & 16(44.4) \\ 2 & 7(19.4) \\ \text { Previous allo-HSCT } & 16(44.4) \\ \quad \text { Yes } & 20(55.6) \\ \text { No } & \\ \text { FLAG-Ida prior to FLAG-Eto } & 11(30.6) \\ \quad \text { Yes } & 25(69.4) \\ \text { No } & \end{array}$

Data are presented as $n(\%)$, mean $\pm \mathrm{SD}$, or median (range), as appropriate. allo-HSCT, allogeneic hematopoietic stem cell transplantation; $\mathrm{BM}$, bone marrow; $\mathrm{BMD}$, bone marrow disease; CCI, Charlson Comorbidity Index; CR, complete remission; CRi, CR with incomplete hematologic recovery; ECOG, Eastern Cooperative Oncology Group; Hb, hemoglobin; LDH, lactate dehydrogenase; PIF, primary induction failure; Plt, platelets; PS, performance status; $\mathrm{SD}$, standard deviation; WBC, white blood cell count. ${ }^{a}$ prior to 1 st cycle of FLAG-Eto. ${ }^{b}$ duration of last CR/CRi prior to FLAG-Eto, only measured in patients who achieved a CR/ CRi prior to FLAG-Eto.

$(n=13)$ of patients treated according to the FLAG-Eto protocol experienced at least one prior relapse and correspondingly the median number of prior salvage therapies was 1 (range $0-2$ ). These salvage therapies comprised intensive chemotherapy protocols; 4 patients received hypomethylating agents. Further, $44 \%(n=16)$ of FLAGEto-treated patients previously underwent allogeneic stem cell transplantation. In line with the number of preceding relapses and intensive treatments, patients receiving FLAG-Eto showed an impaired performance status with $11.1 \%(n=4)$ of patients having an ECOG status of $\geq 3$.

\section{Flag-Eto Shows Anti-Leukemic Activity in Heavily \\ Pretreated Relapsed/Refractory AML Patients}

Nine patients $(25.7 \%)$ receiving the FLAG-Eto protocol achieved CR, 5.7\% CRi $(n=2), 2.9 \%(n=1)$ MLFS, $5.7 \%(n=2)$ CRi/MLFS, and 5.7\% $(n=2)$ partial remission (Fig. 1a). This resulted in a 1-year disease-free survival of $40 \%$ (95\% CI 9.6-70.3) with a median disease-free survival of 8 months (95\% CI 0.6-15.5) for CR/CRi/MLFS 


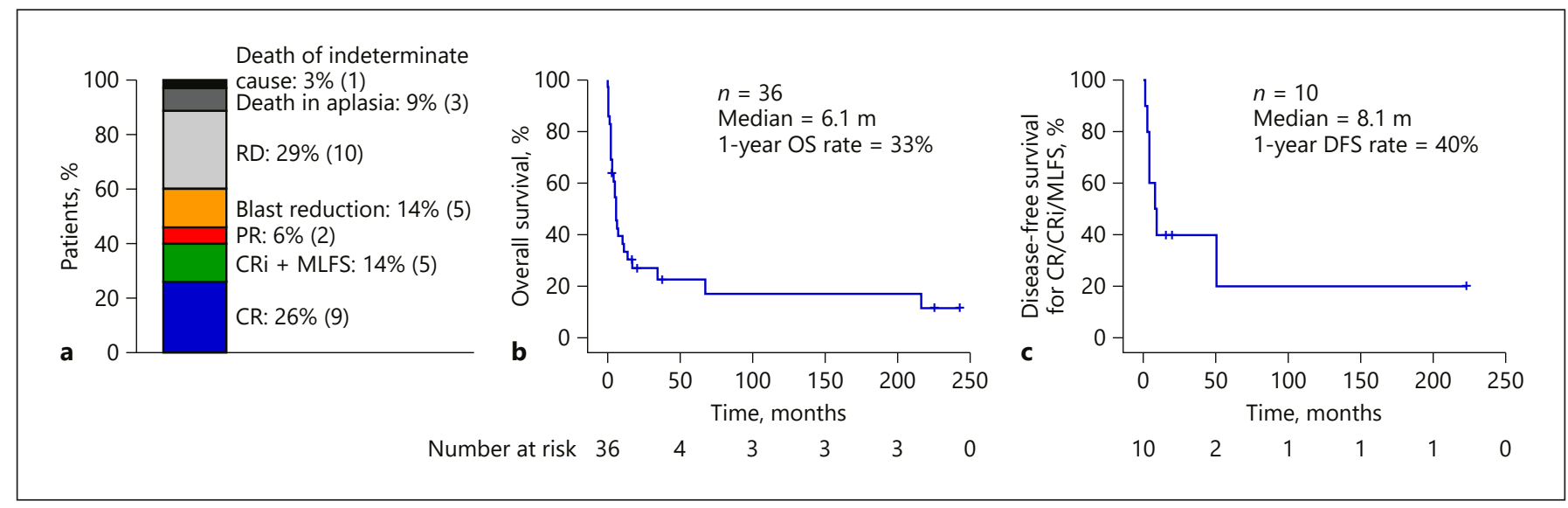

Fig. 1. Response rates (a), and Kaplan-Meier curves for disease-free survival (b) and overall survival (c) for FLAGEto-treated patients. OS, overall survival; DFS, disease-free survival; CR, complete remission; CRi, CR with incomplete hematological recovery; MLFS, morphologic leukemia-free state; PR, partial remission; RD, refractory disease.

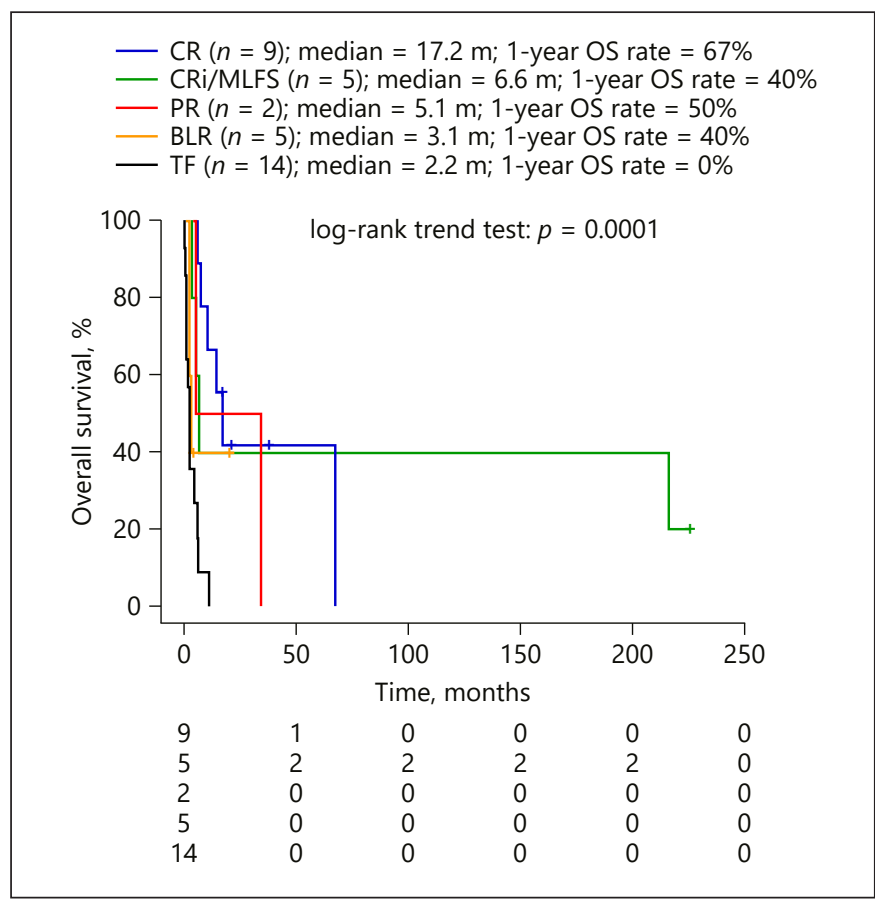

Fig. 2. Kaplan-Meier curves for overall survival subdivided by remission state. CR, complete remission; CRi, CR with incomplete hematological recovery; MLFS, morphologic leukemia-free state; $\mathrm{PR}$, partial remission; BLR, blast reduction; TF, treatment failure.

patients (Fig. 1b). The median overall survival for all treated patients was 6 months (95\% CI 4.5-7.7) with an overall survival rate at 1 year of $33 \%$ (95\% CI 17.6-49.4) considering a median follow-up time of 38 months (range

FLAG-Etoposide for Multiple Relapsed/ Refractory AML
4-246; Fig. 1c). As expected, overall survival depended on remission status with a median of 17.2 months (95\% CI 3-25.5) for patients achieving CR (Fig. 2; online suppl. Tables S1-3; for all online suppl. material, see www.karger.com/doi/10.1159/000503056).

The response to FLAG-Eto treatment did not depend on age; patients over 60 years of age showed CR/CRi/ MLFS rates of $36 \%(n=4 / 11)$ compared to $42 \%(n=$ $10 / 24)$ in patients $\leq 60$ years with a comparable median overall survival for both groups (6.2 vs. 6.1 months for patients $>60$ vs. $\leq 60$ years; online suppl. Tables S1, S2). It remains elusive to what extent the number of prior salvage therapies influenced the response to treatment. Of note, $44.4 \%$ of FLAG-Eto-treated patients received 1 prior salvage treatment and $19.4 \%$ received 2 . While $61.5 \%$ $(n=8 / 13)$ of patients who received FLAG-Eto as a first salvage treatment achieved CR/CRi/MLFS, this rate decreased to $40 \%(n=6 / 15)$ among patients who received FLAG-Eto as a second salvage regimen, and finally none of the 7 patients with 2 prior salvage therapies achieved CR/CRi/MLFS ( $p=0.01$; online suppl. Table S1). However, in this limited cohort size disease-free and overall survival did not differ significantly in dependence of the number of prior salvage therapies (online suppl. Fig. S1). Focusing on patients who relapsed after preceding allogeneic stem cell transplantation, $25 \%(n=4 / 16)$ of patients treated with the FLAG-Eto protocol still achieved CR/ CRi/MLFS (online suppl. Table S1) with a median overall survival for CR/CRi/MLFS patients of 5 months (95\% CI 0-46.8) compared to 14 months (95\% CI 5.1-23.4) in not previously transplanted patients. In conclusion, FLAG- 
Table 2. Documented infections in FLAG-Eto-treated patients $(n=36)$

\begin{tabular}{ccc}
\hline & $n(\%)$ & $\mathrm{n} / \mathrm{a}$ \\
\hline Infection $_{\text {FUO }}$ & $34(94.4)$ & 0 \\
Diarrhea $^{\mathrm{a}}$ & $2(6.1)$ & 3 \\
Mucositis $^{\mathrm{a}}$ & $4(11.1)$ & 0 \\
Pneumonia & $6(16.7)$ & 0 \\
$\quad$ Grade 4 & $2(5.6)$ & 0 \\
Grade 5 & $2(5.6)$ & \\
Sepsis & & \\
Grade 4 & $2(5.6)$ & 0 \\
Grade 5 & $3(8.3)$ & \\
\hline
\end{tabular}

CTC, common toxicity criteria; FUO, fever of unknown origin; n/a, not available. ${ }^{a}$ Any CTC grade.

Eto showed significant anti-leukemic activity which did not depend on age but correlated with the number of preceding salvage therapies.

\section{Toxicity Profile of FLAG-Eto Salvage Therapy}

Nearly all patients experienced grade 3-4 myelosuppression with a median duration until neutrophil recovery $(\geq 0.5 \mathrm{~nL})$ of 24.5 days (range 19-30). Patients treated with FLAG-Eto received a median of 8 (range $0-20$ ) red blood cell units and 9 (range 3-33) platelet units; 5.7\% $(n=2)$ suffered from grade 3 bleeding complications and almost all patients experienced infections of any severity according to CTCAE (Table 2). Four FLAG-Eto-treated patients (11.1\%) got grade $4 / 5$ pneumonia and $13.8 \%$ $(n=5)$ had sepsis, of whom $8.3 \%(n=3)$ died (Table 2$)$. Considering other organ functions, only few cases with higher-grade toxicities were observed (Table 3 ). The median duration of hospitalization reached 38.5 days (range $27-90)$. The 30 -day mortality was $8.3 \%(n=3)$ and increased to $16.7 \%(n=6)$ at day 60 . Cause of death during the first 30 days after treatment initiation was sepsis $(66 \%$, $n=2)$ and resistant disease with meningeal infiltration $(33 \%, n=1)$. Within the following 30 days, 2 further patients succumbed to infectious complications and 1 patient had relapse. Importantly, there were no significant differences in higher-grade toxicity in dependence of age or number of prior salvage therapies.

\section{Post-Remission Treatment}

We have recently confirmed allogeneic stem cell transplantation as the consolidation treatment of choice for first-time relapsed/refractory AML patients to achieve
Table 3. Nonhematological grades 3 and 4 toxicities according to the common toxicity criteria

\begin{tabular}{llll}
\hline & \multicolumn{3}{l}{ FLAG-Eto $(n=36)$} \\
\cline { 2 - 4 } & grade 3 & grade 4 & assessed \\
\hline Nephro- and hepatotoxicity & & & \\
Creatinine & $2(6.5)$ & $0(0)$ & 31 \\
Bilirubin & $2(6.5)$ & $0(0)$ & 31 \\
AP & $0(0)$ & $0(0)$ & 21 \\
GGT & $7(23.3)$ & $0(0)$ & 30 \\
AST & $6(19.4)$ & $0(0)$ & 31 \\
ALT & $4(12.9)$ & $0(0)$ & 31 \\
Cardiotoxicity & & & \\
Arrhythmia & $0(0)$ & $1(2.8)$ & 35 \\
Myocardial infarction & $0(0)$ & $0(0)$ & 35 \\
EF reduction abs. ${ }^{a, c}$ & $1(8.3)$ & $0(0)$ & 12 \\
EF reduction diff. ${ }^{b, c}$ & $0(0)$ & $0(0)$ & 5 \\
\hline
\end{tabular}

Data are presented as $n(\%)$. AP, alkaline phosphatase; ALT, alanine aminotransferase; AST, aspartate aminotransferase; EF, ejection fraction; GGT, gamma-glutamyltransferase; n/a, not available. ${ }^{a} \mathrm{EF}$ reduction absolute. ${ }^{\mathrm{b}} \mathrm{EF}$ reduction in difference (in cases where EF before Flag-Eto was available). ${ }^{c}$ EF reduction in sepsis not included.

long-lasting relapse-free survival [9]. We next evaluated post-remission treatment among those patients who achieved at least partial remission after FLAG-Eto treatment. In fact, the majority of patients could continue with consolidation therapy: $33 \%(5 / 15)$ of the evaluable patients underwent allogeneic stem cell transplantation, $27 \%(n=4)$ received donor lymphocytes, and $13 \%(n=2)$ were consolidated with chemotherapy. The low number of patients in each consolidation treatment subgroup did not allow firm conclusions on the prognostic value of each therapy modality in multiple relapsed/refractory patients. However, it appears that any consolidation therapy positively affected overall survival (online suppl. Fig. S2, Tables S2-3).

\section{Discussion}

Given the high rate of multiple occurring relapse and refractoriness to chemotherapy in AML, different salvage protocols are urgently needed. The requirements for such therapies, apart from high efficacy, are good tolerability with maintenance of good performance status to enable any consolidating intensive treatment. Frequently, cumulative anthracycline dosages have been reached in multiple relapsed/refractory AML patients which built 


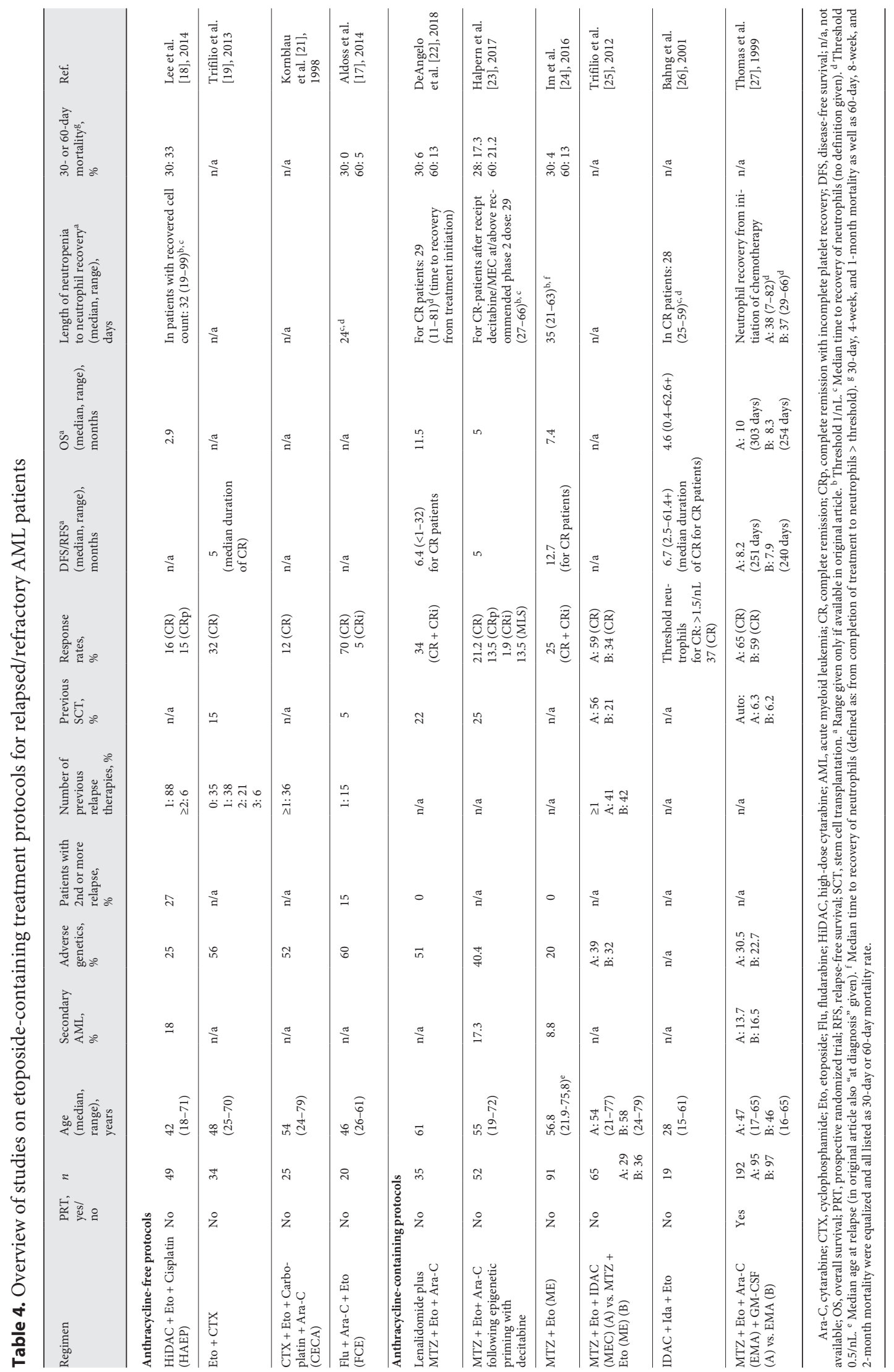


the rationale to introduce the here presented FLAG-Eto protocol for patients not eligible for further anthracycline treatment. In this heavily pretreated patient cohort $25.7 \%$ achieved CR and 14.3\% CRi/MLFS with a 1-year disease free survival of $40 \%$. Noteworthy, long-term outcome for patients who achieved remission under FLAG-Eto was inferior compared to long-term survival data of our recently published cohort of FLAG-Ida-treated mainly first-time relapsed/primary refractory AML patients [9]. These obvious differences in the quality of remission are most likely not due to the chemotherapeutic regimen itself but rather rely on the number of prior relapses and the biology of disease. This is supported by the here presented decreasing response rates along with the increasing number of preceding salvage therapies. Despite the impaired performance status among FLAG-Eto-treated patients at baseline we did not observe a significant increase in the frequency of grade $3 / 4$ toxicities compared to the toxicity profile of our previously published FLAGIda regime [9].

To our knowledge, there is only one published study on the efficacy of the FLAG-Eto protocol in 20 patients, of whom only $15 \%$ received one prior salvage therapy and $5 \%$ previously underwent allogeneic stem cell transplantation [17]. In this patient cohort higher anti-leukemic activity with CR/CRi rates of $75 \%$ and a 1 -year overall survival of $71 \%$ has been achieved with a more beneficial toxicity profile. These results raise the question of the role of the FLAG-Eto protocol for up-front salvage treatment. Focusing on studies which applied salvage therapies in multiple relapsed/refractory AML patients, CR or CR/ CRp (CR with incomplete platelet counts) was achieved in $31-36 \%$ of patients with a median overall survival of about 3 months (Table 4) [18-20], which suggests that remission rates achieved with the FLAG-Eto regimen range above average. However, randomized trials are required to define optimal chemotherapeutic combinations for different subsets of relapsed/refractory AML.

In conclusion, FLAG-Eto for multiple relapsed/refractory AML patients is an effective salvage regimen, also eligible for selected elderly patients. The impaired performance status and higher comorbidity index of heavily pretreated patients at baseline were not worsened through FLAG-Eto. This regimen still enabled implementation of further consolidation therapies such as allogeneic stem cell transplantation.

\section{Acknowledgments}

The authors would like to thank Nils Lehmann, Institute of Medical Informatics, Biometry and Epidemiology, University Hospital Essen, University Duisburg-Essen, for consultation on statistical analyses.

\section{Statement of Ethics}

The study was approved by the relevant Ethics Committee and followed guidelines set out in the Declaration of Helsinki.

\section{Disclosure Statement}

The authors have no conflicts of interest to declare.

\section{Author Contributions}

J.W., R.N., C.S., M.F., U.D., and M.H. collected and analyzed the data. J.W. and M.H. wrote the manuscript.

\section{References}

1 Büchner T, Schlenk RF, Schaich M, Döhner K, Krahl R, Krauter J, et al. Acute Myeloid Leukemia (AML): different treatment strategies versus a common standard arm-combined prospective analysis by the German AML Intergroup. J Clin Oncol. 2012 Oct;30(29):3604-10.

2 Tiong IS, Wei AH. New drugs creating new challenges in acute myeloid leukemia. Genes Chromosomes Cancer. 2019 Mar;gcc.22750.

3 Bergua JM, Montesinos P, Martinez-Cuadrón D, Fernández-Abellán P, Serrano J, Sayas MJ, et al.; PETHEMA group. A prognostic model for survival after salvage treatment with FLAG-Ida +/- gemtuzumab-ozogamicine in adult patients with refractory/relapsed acute myeloid leukaemia. Br J Haematol. 2016 Sep; 174(5):700-10.
4 Thiel A, Schetelig J, Pönisch W, SchäferEckart K, Aulitzky W, Peter N, et al.; East German Study Group Hematology/Oncology (OSHO); Study Alliance Leukemia (SAL). Mito-FLAG with Ara-C as bolus versus continuous infusion in recurrent or refractory AML-long-term results of a prospective randomized intergroup study of the East German Study Group Hematology/Oncology (OSHO) and the Study Alliance Leukemia (SAL). Ann Oncol. 2015 Jul;26(7): 1434-40.

5 Wierzbowska A, Robak T, Pluta A, Wawrzyniak E, Cebula B, Hołowiecki J, et al.; Polish Adult Leukemia Group. Cladribine combined with high doses of arabinoside cytosine, mitoxantrone, and G-CSF (CLAG-
M) is a highly effective salvage regimen in patients with refractory and relapsed acute myeloid leukemia of the poor risk: a final report of the Polish Adult Leukemia Group. Eur J Haematol. 2008 Feb;80(2): 115-26.

6 Ferrara F, Melillo L, Montillo M, Leoni F, Pinto A, Mele G, et al. Fludarabine, cytarabine, and G-CSF (FLAG) for the treatment of acute myeloid leukemia relapsing after autologous stem cell transplantation. Ann Hematol. 1999 Aug;78(8):380-4.

7 Montillo M, Mirto S, Petti MC, Latagliata R, Magrin S, Pinto A, et al. Fludarabine, cytarabine, and G-CSF (FLAG) for the treatment of poor risk acute myeloid leukemia. Am J Hematol. 1998 Jun;58(2):105-9. 
8 Visani G, Tosi P, Zinzani PL, Manfroi S, Ottaviani E, Testoni N, et al. FLAG (fludarabine + high-dose cytarabine + G-CSF): an effective and tolerable protocol for the treatment of 'poor risk' acute myeloid leukemias. Leukemia. 1994 Nov;8(11):1842-6.

9 Westhus J, Noppeney R, Duhrsen U, Hanoun M. FLAG salvage therapy combined with idarubicin in relapsed/refractory acute myeloid leukemia. Leuk Lymphoma. 2019 Apr;60(4): 1014-22.

10 Grimwade D, Walker H, Oliver F, Wheatley K, Harrison C, Harrison G, et al.; The Medical Research Council Adult and Children's Leukaemia Working Parties. The importance of diagnostic cytogenetics on outcome in AML: analysis of 1,612 patients entered into the MRC AML 10 trial. Blood. 1998 Oct;92(7): 2322-33.

11 Döhner H, Estey E, Grimwade D, Amadori S, Appelbaum FR, Büchner T, et al. Diagnosis and management of AML in adults: 2017 ELN recommendations from an international expert panel. Blood. 2017 Jan;129(4):424-47.

12 Döhner H, Estey EH, Amadori S, Appelbaum FR, Büchner T, Burnett AK, et al.; European LeukemiaNet. Diagnosis and management of acute myeloid leukemia in adults: recommendations from an international expert panel, on behalf of the European LeukemiaNet. Blood. 2010 Jan;115(3):453-74.

13 Byrd JC, Mrózek K, Dodge RK, Carroll AJ, Edwards CG, Arthur DC, et al. Pretreatment cytogenetic abnormalities are predictive of induction success, cumulative incidence of relapse, and overall survival in adult patients with de novo acute myeloid leukemia: results from Cancer and Leukemia Group B (CALGB 8461). Blood. 2002 Dec;100(13):4325-36.

14 Slovak ML, Kopecky KJ, Cassileth PA, Harrington $\mathrm{DH}$, Theil KS, Mohamed A, et al. Karyotypic analysis predicts outcome of preremission and postremission therapy in adult acute myeloid leukemia: a Southwest Oncology Group/Eastern Cooperative Oncology Group Study. Blood. 2000 Dec;96(13):407583.
15 National Cancer Institute. Common terminology criteria for adverse events (CTCAE) version 4.0. 2009 [cited 2017 March 21]. Available from: https://www.eortc.be/services/doc/ctc/ctcae_4.03_2010-06-14_quickreference_5x7.pdf.

16 Cheson BD, Bennett JM, Kopecky KJ, Büchner T, Willman CL, Estey EH, et al.; International Working Group for Diagnosis, Standardization of Response Criteria, Treatment Outcomes, and Reporting Standards for Therapeutic Trials in Acute Myeloid Leukemia. Revised recommendations of the International Working Group for Diagnosis, Standardization of Response Criteria, Treatment Outcomes, and Reporting Standards for Therapeutic Trials in Acute Myeloid Leukemia. J Clin Oncol. 2003 Dec;21(24):4642-9.

17 Aldoss I, Ji L, Haider M, Pullarkat V. The combination of fludarabine, cytarabine and etoposide is an active and well-tolerated regimen in relapsed/refractory acute myeloid leukemia. Acta Haematol. 2014;131(4):202-7.

18 Lee YG, Kwon JH, Kim I, Yoon SS, Lee JS, Park S. Effective salvage therapy for high-risk relapsed or refractory acute myeloid leukaemia with cisplatin in combination with highdose cytarabine and etoposide. Eur J Haematol. 2014 Jun;92(6):478-84.

19 Trifilio S, Zhou Z, Altman J, Frankfurt O, Pantiru M, Mehta J. Dose-intense etoposidecyclophosphamide without stem cell transplantation for patients with intermediate and high cytogenetic risk primary refractory and relapsed acute myeloid leukemia. Leuk Res. 2013 Aug;37(8):872-6.

20 Estey E, Plunkett W, Gandhi V, Rios MB, Kantarjian H, Keating MJ. Fludarabine and arabinosylcytosine therapy of refractory and relapsed acute myelogenous leukemia. Leuk Lymphoma. 1993 Mar;9(4-5):343-50.

21 Kornblau SM, Kantarjian H, O’Brien S, Andreeff M, Koller CA, Beran M, et al. CECAcyclophosphamide, etoposide, carboplatin and cytosine arabinoside- a new salvage regimen for relapsed or refractory acute myelogenous leukemia. Leuk Lymphoma. 1998 Jan; 28(3-4):371-5.
22 DeAngelo DJ, Brunner AM, Werner L, Avigan D, Fathi AT, Sperling AS, et al. A phase I study of lenalidomide plus chemotherapy with mitoxantrone, etoposide, and cytarabine for the reinduction of patients with acute myeloid leukemia. Am J Hematol. 2018 Feb; 93(2):254-61.

23 Halpern AB, Othus M, Huebner EM, Buckley SA, Pogosova-Agadjanyan EL, Orlowski KF, et al. Mitoxantrone, etoposide and cytarabine following epigenetic priming with decitabine in adults with relapsed/refractory acute myeloid leukemia or other high-grade myeloid neoplasms: a phase 1/2 study. Leukemia. 2017 Dec;31(12):2560-7.

24 Im A, Amjad A, Agha M, Raptis A, Hou JZ, Farah R, et al. Mitoxantrone and Etoposide for the Treatment of Acute Myeloid Leukemia Patients in First Relapse. Oncol Res. 2016; 24(2):73-80.

25 Trifilio SM, Rademaker AW, Newman D, Coyle K, Carlson-Leuer K, Mehta J, et al. Mitoxantrone and etoposide with or without intermediate dose cytarabine for the treatment of primary induction failure or relapsed acute myeloid leukemia. Leuk Res. 2012 Apr;36(4): 394-6.

26 Bahng H, Lee JH, Ahn JH, Lee JH, Lee JS, Kim $\mathrm{SH}$, et al. Combination chemotherapy utilizing continuous infusion of intermediate-dose cytarabine for refractory or recurrent acute myeloid leukemia. Leuk Res. 2001 Mar;25(3): 213-6.

27 Thomas X, Fenaux P, Dombret H, Delair S, Dreyfus F, Tilly H, et al. Granulocyte-macrophage colony-stimulating factor (GMCSF) to increase efficacy of intensive sequential chemotherapy with etoposide, mitoxantrone and cytarabine (EMA) in previously treated acute myeloid leukemia: a multicenter randomized placebo-controlled trial (EMA91 Trial). Leukemia. 1999 Aug;13(8):1214-20. 